\title{
LOGARITHMIC MEANS OF WALSH-FOURIER SERIES
}

\author{
USHANGI GOGINAVA
}

Received 11 October, 2018

\begin{abstract}
In this paper we discuss some convergence and divergence properties of subsequences of logarithmic means of Walsh-Fourier series. We give necessary and sufficient conditions for the convergence regarding logarithmic variation of numbers.
\end{abstract}

2010 Mathematics Subject Classification: 42C10

Keywords: Walsh-Fourier series, Nörlund Logarithmic means, almost everywhere converges, convergence in norm

\section{WALSH FUNCTIONS}

We shall denote the set of all non-negative integers by $\mathbb{N}$, the set of all integers by $\mathbb{Z}$ and the set of dyadic rational numbers in the unit interval $\mathbb{I}:=[0,1)$ by $\mathbb{Q}$. In particular, each element of $\mathbb{Q}$ has the form $\frac{p}{2^{n}}$ for some $p, n \in \mathbb{N}, 0 \leq p \leq 2^{n}$.

Denote the dyadic expension of $n \in \mathbb{N}$ and $x \in \mathbb{I}$ by

$$
n=\sum_{j=0}^{\infty} \varepsilon_{j}(n) 2^{j}, \varepsilon_{j}(n)=0,1
$$

and

$$
x=\sum_{j=0}^{\infty} \frac{x_{j}}{2^{j+1}}, x_{j}=0,1 .
$$

In the case of $x \in \mathbb{Q}$ chose the expension which terminates in zeros. Define the dyadic addition + as

$$
x+y=\sum_{k=0}^{\infty}\left|x_{k}-y_{k}\right| 2^{-(k+1)} .
$$

The sets $I_{n}(x):=\left\{y \in \mathbb{I}: y_{0}=x_{0}, \ldots, y_{n-1}=x_{n-1}\right\}$ for $x \in \mathbb{I}, I_{n}:=I_{n}(0)$ for $0<n \in \mathbb{N}$ and $I_{0}(x):=\mathbb{I}$ are the dyadic intervals of $\mathbb{I}$. For $0<n \in \mathbb{N}$ denote by $|n|:=\max \left\{j \in \mathbb{N}: n_{j} \neq 0\right\}$, that is, $2^{|n|} \leq n<2^{|n|+1}$.

The author supported by Shota Rustaveli National Science Foundation grant 217282 (Operators of Fourier analysis in some classical and new function spaces ). 
The Rademacher system is defined by

$$
\rho_{n}(x):=(-1)^{x_{n}} \quad(x \in \mathbb{I}, n \in \mathbb{N}) .
$$

The Walsh-Paley system is defined as the sequence of the Walsh-Paley functions:

$$
w_{n}(x):=\prod_{k=0}^{\infty}\left(\rho_{k}(x)\right)^{n_{k}}=(-1)^{\sum_{k=0}^{|n|} n_{k} x_{k}}(x \in \mathbb{I}, n \in \mathbb{N}) .
$$

The Walsh-Dirichlet kernel is defined by

$$
D_{n}(x)=\sum_{k=0}^{n-1} w_{k}(x)(n \in \mathbb{N}), D_{0}=0 .
$$

Recall that (see [20])

$$
D_{2^{n}}(x)=\left\{\begin{array}{c}
2^{n}, \text { if } x \in I_{n}(0) \\
0, \text { if } x \in \mathbb{I} \backslash I_{n}(0)
\end{array} .\right.
$$

As usual, denote by $L_{1}$ (II) the set of measurable functions defined on $\mathbb{I}$, for which

$$
\|f\|_{1}:=\int_{\mathbb{I}}|f(t)| d t<\infty
$$

Let $f \in L_{1}$ (II). The partial sums of the Walsh-Fourier series are defined as follows:

$$
S_{M}(x, f):=\sum_{i=0}^{M-1} \widehat{f}(i) w_{i}(x)
$$

where the number

$$
\widehat{f}(i)=\int_{\mathbb{I}} f(t) w_{i}(t) d t
$$

is said to be the $i$ th Walsh-Fourier coefficient of the function $f$. Set $E_{n}(x, f)=$ $S_{2^{n}}(x, f)$. The maximal function is defined by

$$
E^{*}(x, f)=\sup _{n \in \mathbb{N}} E_{n}(x,|f|) .
$$

The notiation $a \lesssim b$ in the proofs stands for $a<c \cdot b$, where $c$ is an absolute constant. 


\section{LOGARITHMIC MEANS}

In the literature, there is the notion of Riesz's logarithmic means of a Fourier series. The $n$-th Riesz's logarithmic means of the Fourier series of an integrable function $f$ is defined by

$$
R_{n}(x, f):=\frac{1}{l_{n}} \sum_{k=1}^{n} \frac{S_{k}(x, f)}{k},
$$

where $l_{n}:=\sum_{k=1}^{n}(1 / k)$.

Riesz's logarithmic means with respect to the trigonometric system was studied by a lot of authors. This means with respect to the Walsh and Vilenkin systems was discussed by Simon [21], Blahota, Gát [1], Gát [4], Gát, Goginava [8].

Let $\left\{q_{k}: k \geq 0\right\}$ be a sequence of nonnegative numbers. The $n$-th Nörlund means for the Fourier series of $f$ is defined by

$$
\frac{1}{Q_{n}} \sum_{k=0}^{n-1} q_{n-k} S_{k}(f),
$$

where

$$
Q_{n}:=\sum_{k=1}^{n} q_{k} .
$$

If $q_{k}=k$, then we get the Nörlund logarithmic means

$$
t_{n}(x, f):=\frac{1}{l_{n}} \sum_{k=0}^{n-1} \frac{S_{k}(x, f)}{n-k} .
$$

In this paper we call it logarithmic mean altough, it is a kind of "reverse" Reisz's logarithmic mean.

It is easy to see that

$$
t_{n}(x, f)=\int_{\mathbb{I}} f(t) F_{n}(x+t) d t,
$$

where by $F_{n}(t)$ we denote $n$th logarithmic kernel, i. e.

$$
F_{n}(t):=\frac{1}{l_{n}} \sum_{k=0}^{n-1} \frac{D_{k}(t)}{n-k} .
$$

and Fejér kernel is defined by

$$
K_{n}(t):=\frac{1}{n} \sum_{k=1}^{n} D_{k}(t) .
$$




\section{3. $L_{1}$ - ESTIMATION FOR LOGARITHMIC KERNEL}

For $n=\sum_{j=0}^{\infty} \varepsilon_{j}(n) 2^{j}, \varepsilon_{j}(n)=0,1$ we define

$$
n(k):=\sum_{j=0}^{k} \varepsilon_{j}(n) 2^{j} .
$$

It is easy to see that $n(|n|)=n$. In this paper for $L_{1}$-norm of logarithmic means we prove the following two sides estimation.

Theorem 1. Let $n \in \mathbb{N}$. Then

$$
\left\|\frac{1}{l_{n}} \sum_{j=1}^{n} \frac{D_{n-j}}{j}\right\|_{1} \sim \frac{1}{|n|} \sum_{k=1}^{|n|}\left|\varepsilon_{k}(n)-\varepsilon_{k+1}(n)\right| l_{n(k-1)} .
$$

Proof. We can write

$$
\begin{aligned}
& \sum_{j=1}^{n} \frac{D_{n-j}(t)}{j} \\
& =\sum_{j=1}^{n(|n|-1)} \frac{D_{n-j}(t)}{j}+\sum_{j=n(|n|-1)+1}^{n} \frac{D_{n-j}(t)}{j} \\
& =\sum_{j=1}^{n(|n|-1)} \frac{D_{\varepsilon_{|n|}(n) 2^{|n|}+n(|n|-1)-j}(t)}{j}+\sum_{j=n(|n|-1)+1}^{n} \frac{D_{n-j}(t)}{j} .
\end{aligned}
$$

Since

$$
D_{\varepsilon_{|n|}(n) 2^{|n|}+n(|n|-1)-j}(t)=\varepsilon_{|n|}(n) D_{2^{|n|}}(t)+\left(w_{2^{|n|}}(t)\right)^{\varepsilon_{|n|}(n)} D_{n(|n|-1)-j}(t),
$$

from (3.1) we have

$$
\begin{aligned}
& \sum_{j=1}^{n} \frac{D_{n-j}(t)}{j} \\
& =\varepsilon_{|n|}(n) D_{2^{|n|}}(t) l_{n(|n|-1)} \\
& \quad+\left(w_{2^{|n|}}(t)\right)^{\varepsilon_{|n|}(n)} \sum_{j=1}^{n(|n|-1)} \frac{D_{n(|n|-1)-j}(t)}{j} \\
& \quad+\varepsilon_{|n|}(n) \sum_{j=1}^{2^{|n|}-1} \frac{D_{2^{|n|}-j}(t)}{j+n(|n|-1)} .
\end{aligned}
$$


Iterating this equality we obtain

$$
\begin{aligned}
& \sum_{j=1}^{n} \frac{D_{n-j}(t)}{j} \\
& =\left(\sum_{j=2}^{|n|} \varepsilon_{j}(n) D_{2^{j}}(t) l_{n(j-1)}\right) \prod_{k=j+1}^{|n|}\left(\rho_{k}(t)\right)^{\varepsilon_{k}(n)} \\
& \quad+\left(\sum_{j=2}^{|n|} \varepsilon_{j}(n) \sum_{k=1}^{2^{j}-1} \frac{D_{2^{j}-k}(t)}{k+n(j-1)}\right) \prod_{s=j+1}^{|n|}\left(\rho_{S}(t)\right)^{\varepsilon_{S}(n)} \\
& \quad+\left(\sum_{j=1}^{n(1)} \frac{D_{n(1)-j}(t)}{j}\right) \prod_{k=2}^{|n|}\left(\rho_{k}(t)\right)^{\varepsilon_{k}(n)}
\end{aligned}
$$

Since

$$
\varepsilon_{j}(n) D_{2^{j}}(t) \prod_{k=0}^{j}\left(\rho_{k}(t)\right)^{\varepsilon_{k}(n)}=\varepsilon_{j}(n) D_{2^{j}}(t) \rho_{j}(t)
$$

we have

$$
\begin{aligned}
& \left(\sum_{j=2}^{|n|} \varepsilon_{j}(n) D_{2^{j}}(t) l_{n(j-1)}\right) \prod_{k=j+1}^{|n|}\left(\rho_{k}(t)\right)^{\varepsilon_{k}(n)} \\
& =w_{n}(t)\left(\sum_{j=2}^{|n|} \varepsilon_{j}(n) D_{2^{j}}(t) l_{n(j-1)}\right) \prod_{k=0}^{j}\left(\rho_{k}(t)\right)^{\varepsilon_{k}(n)} \\
& =w_{n}(t)\left(\sum_{j=2}^{|n|} \varepsilon_{j}(n) D_{2^{j}}(t) \rho_{j}(t) l_{n(j-1)}\right) .
\end{aligned}
$$


Combining (3.2) and (3.3) we conclude that

$$
\begin{aligned}
\sum_{j=1}^{n} \frac{D_{n-j}(t)}{j} \\
=w_{n}(t)\left(\sum_{j=2}^{|n|} \varepsilon_{j}(n) D_{2^{j}}(t) \rho_{j}(t) l_{n(j-1)}\right) \\
\quad+\left(\sum_{j=2}^{|n|} \varepsilon_{j}(n) \sum_{k=1}^{2^{j}-1} \frac{D_{2^{j}-k}(t)}{k+n(j-1)}\right) \prod_{s=j+1}^{|n|}\left(\rho_{s}(t)\right)^{\varepsilon_{s}(n)} \\
+\left(\sum_{j=1}^{n(1)-1} \frac{D_{n(1)-j}(t)}{j}\right) \prod_{k=2}^{|n|}\left(\rho_{k}(t)\right)^{\varepsilon_{k}(n)} \\
=: H_{n}^{(1)}(t)+H_{n}^{(2)}(t)+H_{n}^{(3)}(t) .
\end{aligned}
$$

Since (see [9])

$$
D_{2^{j}-k}(t)=D_{2^{j}}(t)-w_{2^{j}-1}(t) D_{k}(t), k=1,2, \ldots, 2^{j}-1
$$

for $H_{n}^{(2)}(t)$ we can write

$$
\begin{aligned}
H_{n}^{(2)}(t) & \left(\sum_{j=2}^{|n|} \varepsilon_{j}(n) D_{2^{j}}(t) \sum_{k=1}^{2^{j}-1} \frac{1}{k+n(j-1)}\right) \prod_{s=j+1}^{|n|}\left(\rho_{s}(t)\right)^{\varepsilon_{S}(n)} \\
& -\left(\sum_{j=2}^{|n|} \varepsilon_{j}(n) w_{2^{j}-1}(t) \sum_{k=1}^{2^{j}-1} \frac{D_{k}(t)}{k+n(j-1)}\right) \prod_{s=j+1}^{|n|}\left(\rho_{s}(t)\right)^{\varepsilon_{S}(n)} \\
= & : H_{n}^{(21)}(t)+H_{n}^{(22)}(t) .
\end{aligned}
$$

Since

$$
\begin{aligned}
H_{n}^{(21)}(t)= & \left(\sum_{j=2}^{|n|} \varepsilon_{j}(n) D_{2^{j}}(t)\left(l_{n(j)-1}-l_{n(j-1)}\right)\right) \\
& \times \prod_{s=j+1}^{|n|}\left(\rho_{s}(t)\right)^{\varepsilon_{S}(n)}
\end{aligned}
$$

from (1.1) we get

$$
\left\|H_{n}^{(21)}\right\|_{1} \leq \sum_{j=2}^{|n|} \varepsilon_{j}(n)\left(l_{n(j)}-l_{n(j-1)}\right) \leq c|n| .
$$


Usin Abel's transformation we obtain

$$
\begin{aligned}
& \sum_{k=1}^{2^{j}-1} \frac{D_{k}(t)}{k+n(j-1)} \\
& =\sum_{k=1}^{2^{j}-2}\left(\frac{1}{k+n(j-1)}-\frac{1}{k+1+n(j-1)}\right) k K_{k}(t) \\
& \quad+\frac{2^{j}-1}{2^{j}-1+n(j-1)} K_{2^{j}-1}(t) .
\end{aligned}
$$

Since (see [20]) $\sup _{n}\left\|K_{n}\right\|_{1}<\infty$ for $H_{n}^{(22)}(t)$ we can write

$$
\begin{aligned}
& \left\|H_{n}^{(22)}\right\|_{1} \\
& \lesssim \sum_{j=2}^{|n|} \varepsilon_{j}(n) \sum_{k=1}^{2^{j}-1}\left(\frac{1}{k+n(j-1)}-\frac{1}{k+1+n(j-1)}\right) k \\
& \quad+\sum_{j=2}^{|n|} \varepsilon_{j}(n) \frac{2^{j}-1}{2^{j}-1+n(j-1)} \\
& \lesssim \sum_{j=2}^{|n|} \varepsilon_{j}(n) \sum_{k=1}^{2^{j}-1} \frac{1}{(k+n(j-1))} \\
& \quad+\sum_{j=2}^{|n|} \varepsilon_{j}(n) \sum_{k=1}^{2^{j}-1} \frac{n(j-1)}{(k+n(j-1))^{2}}+\sum_{j=2}^{|n|} \varepsilon_{j}(n) \frac{2^{j}-1}{2^{j}-1+n(j-1)} \\
& \lesssim \sum_{j=2}^{|n|} \varepsilon_{j}(n)\left(l_{n(j)}-l_{n(j-1)}\right)+|n| \lesssim|n| .
\end{aligned}
$$

Combining (3.5)-(3.7) we conclude that

$$
\left\|H_{n}^{(2)}\right\|_{1} \leq c|n| \text {. }
$$

It is easy to see that

$$
\sup _{n}\left\|H_{n}^{(3)}\right\|_{1} \leq c .
$$

First, we find upper estimation for $\left\|H_{n}^{(1)}\right\|_{1}$. We can write

$$
H_{n}^{(1)}(t)=w_{n}(t)\left(\sum_{j=2}^{|n|} \varepsilon_{j}(n) l_{n(j-1)}\left(D_{2^{j+1}}(t)-D_{2^{j}}(t)\right)\right)
$$




$$
\begin{aligned}
= & w_{n}(t)\left(\sum_{j=2}^{|n|-1}\left(\varepsilon_{j}(n) l_{n(j-1)}-\varepsilon_{j+1}(n) l_{n(j)}\right) D_{2^{j+1}}(t)\right) \\
& +w_{n}(t) l_{n(|n|-1)} D_{2^{|n|+1}}-w_{n}(t) \varepsilon_{2}(n) l_{n(1)} D_{2^{2}}(t) .
\end{aligned}
$$

Hence, from (1.1) we obtain

$$
\begin{aligned}
\left\|H_{n}^{(1)}\right\|_{1} \leq & \sum_{j=2}^{|n|-1}\left|\varepsilon_{j}(n) l_{n(j-1)}-\varepsilon_{j+1}(n) l_{n(j)}\right|+c|n| \\
\leq & \sum_{j=2}^{|n|-1}\left|\varepsilon_{j}(n)-\varepsilon_{j+1}(n)\right| l_{n(j-1)} \\
& +\sum_{j=2}^{|n|-1} \varepsilon_{j+1}(n)\left(l_{n(j)}-l_{n(j-1)}\right)+c|n| \\
\leq & \sum_{j=2}^{|n|}\left|\varepsilon_{j}(n)-\varepsilon_{j+1}(n)\right| l_{n(j-1)}+c|n| .
\end{aligned}
$$

Now, we find lower estimation for $\left\|H_{n}^{(1)}\right\|_{1}$. Let $a_{i}$ and $b_{i}, i=1, \ldots, s$ be strictly increasing sequences, i. e.

$$
0 \leq a_{1} \leq b_{1}<a_{2} \leq b_{2}<\cdots<a_{s} \leq b_{s}<a_{s+1}=\infty
$$

for which

$$
\varepsilon_{j}(n)=\left\{\begin{array}{l}
1, a_{i} \leq j \leq b_{i} \\
0, b_{i}<j<a_{i+1}
\end{array} .\right.
$$

Then it is evident that

$$
b_{j}+1<a_{j+1} .
$$

Set

$$
A_{k}:=\left(\frac{1}{2^{a_{k}+1}}, \frac{1}{2^{a_{k}}}\right), B_{k}:=\left(\frac{1}{2^{b_{k}+2}}, \frac{1}{2^{b_{k}+1}}\right), k=1, \ldots, s .
$$

Let $x \in A_{k}$. Then we can write

$$
\begin{aligned}
\left|H_{n}^{(1)}(t)\right| & =\left|\sum_{j=2}^{|n|} \varepsilon_{j}(n)\left(D_{2^{j+1}}(t)-D_{2^{j}}(t)\right) l_{n(j-1)}\right| \\
& =\mid \sum_{i=1}^{k-1} \sum_{j=a_{i}}^{b_{i}}\left(D_{2^{j+1}}(t)-D_{2^{j}}(t)\right) l_{n(j-1)}
\end{aligned}
$$




$$
\begin{aligned}
& +\sum_{j=a_{k}}^{b_{k}}\left(D_{2^{j+1}}(t)-D_{2^{j}}(t)\right) l_{n(j-1)} \mid \\
= & \left|\sum_{i=1}^{k-1} \sum_{j=a_{i}}^{b_{i}} 2^{j} l_{n(j-1)}-2^{a_{k}} l_{n\left(a_{k}-1\right)}\right| .
\end{aligned}
$$

From (3.11) we can write

$$
\begin{aligned}
\sum_{i=1}^{k-1} \sum_{j=a_{i}}^{b_{i}} 2^{j} l_{n(j-1)} & \leq l_{n\left(b_{k-1}-1\right)} \sum_{i=1}^{k-1}\left(2^{b_{i}+1}-2^{a_{i}}\right) \\
& \leq l_{n\left(b_{k-1}-1\right)} \sum_{i=1}^{k-1}\left(2^{b_{i}+1}-2^{b_{i-1}+1}\right) \\
& \leq 2^{b_{k-1}+1} l_{n\left(b_{k-1}-1\right)} \\
& \leq 2^{b_{k-1}+1} l_{n\left(a_{k}-1\right)}
\end{aligned}
$$

Consequently,

$$
\left|H_{n}^{(1)}(t)\right| \geq 2^{a_{k}} l_{n\left(a_{k}-1\right)}-2^{b_{k-1}+1} l_{n\left(a_{k}-1\right)} \geq 2^{a_{k}-1} l_{n\left(a_{k}-1\right)} .
$$

Integrating on $A_{k}$ we get

$$
\int_{A_{k}}\left|H_{n}^{(1)}(t)\right| d t \geq \int_{A_{k}} 2^{a_{k}-1} l_{n\left(a_{k}-1\right)} d t=\frac{l_{n\left(a_{k}-1\right)}}{4} .
$$

On the interval $B_{k}$ we have

$$
\begin{aligned}
\left|H_{n}^{(1)}(t)\right| & =\left|\sum_{i=1}^{k} \sum_{j=a_{i}}^{b_{i}}\left(D_{2^{j+1}}(t)-D_{2^{j}}(t)\right) l_{n(j-1)}\right| \\
& =\sum_{i=1}^{k} \sum_{j=a_{i}}^{b_{i}} 2^{j} l_{n(j-1)} \geq l_{\left(b_{k}-1\right)} 2^{b_{k}}
\end{aligned}
$$

Hence,

$$
\int_{B_{k}}\left|H_{n}^{(1)}(t)\right| d t \geq \int_{B_{k}} l_{\left(b_{k}-1\right)} 2^{b_{k}} d t=\frac{l_{n\left(b_{k}-1\right)}}{4} .
$$


Since $A_{i}, B_{i}, i=1, \ldots, s$ are pairwise disjoint from (3.12) and (3.13) we have

$$
\begin{aligned}
\int_{\mathbb{I}}\left|H_{n}^{(1)}(t)\right| d t & \geq \sum_{k=1}^{s}\left(\int_{A_{k}}\left|H_{n}^{(1)}(t)\right| d t+\int_{B_{k}}\left|H_{n}^{(1)}(t)\right| d t\right) \\
& \geq \frac{1}{4} \sum_{k=1}^{s}\left(l_{n\left(a_{k}-1\right)}+l_{n\left(b_{k}-1\right)}\right) .
\end{aligned}
$$

Since (see (3.10))

$$
n\left(a_{k}-1\right)=n\left(a_{k}-2\right)
$$

and

$$
\left|\varepsilon_{j}(n)-\varepsilon_{j+1}(n)\right|=\left\{\begin{array}{l}
1, j=a_{k}-1 \text { or } b_{k}, k=1,2, \ldots, s \\
0 \text { otherwise }
\end{array}\right.
$$

we conclude that

$$
\int_{\mathbb{I}}\left|H_{n}^{(1)}(t)\right| d t \geq \frac{1}{4} \sum_{k=1}^{|n|}\left|\varepsilon_{k}(n)-\varepsilon_{k+1}(n)\right| l_{n(k-1)} .
$$

Combining (3.4)-(3.10) and (3.14) we complete the proof of Theorem 1.

\section{Almost EVERYwhere CONVERGENCE OF LOGARITHMiC MEANS}

For a non-negative integer $n$ let us denote

$$
V_{S}(n):=\sum_{i=0}^{\infty}\left|\varepsilon_{i}(n)-\varepsilon_{i+1}(n)\right|+\varepsilon_{0}(n)
$$

and

$$
V_{L}(n):=\frac{1}{|n|} \sum_{k=1}^{|n|}\left|\varepsilon_{k}(n)-\varepsilon_{k+1}(n)\right| l_{n(k-1)} .
$$

It is known that if $n_{j}<n_{j+1}$,

$$
\sup _{j} V_{S}\left(n_{j}\right)<\infty
$$

then a. e. $S_{n_{j}}(f) \rightarrow f$. On the other hand, Konyagin [14] proved that the condition (4.1) is not necessary for a. e. convergence of subsequence of partial sums. Moreover, he gave negative answer to the question of Balashov and proved the validity of the following theorem.

Theorem $\mathbf{K}$ (Konyagin). Suppose $\left\{n_{A}\right\}$ is an increasing sequence of natural numbers, $k_{A}:=\left[\log _{2} n_{A}\right]+1$, and $2^{k_{A}}$ is a divider of $n_{A+1}$ for all $A$. Then $S_{n_{A}}(f) \rightarrow f$ a. e. for any function $f \in L_{1}(\mathbb{I})$. 
For instance, a sequence $\left\{n_{A}\right\}, n_{A}:=2^{A^{2}} \sum_{i=0}^{A} 4^{i}$, such that $\sup _{n_{A}} V\left(n_{A}\right)=\infty$, satisfies the hypotheses of the theorem.

Almost ewerywhere convergence of $\left\{t_{2^{A}}(f): A \geq 1\right\}$ with respect to Walsh-Paley system was studied by author [11]. In particular, the following is proved

Theorem G. Let $f \in L_{1}(\mathbb{I})$. Then $t_{2^{A}}(x, f) \rightarrow f(x)$ as $A \rightarrow \infty$ a. e. $x \in \mathbb{I}$.

Nagy in [18] established a similar result for the Walsh-Kaczmarz system. However, a divergence on the set with positive measure for the whole sequence $\left\{t_{n}(f): n \geq 1\right\}$ was proved by Gát and Goginava [7]. Memić [16] improved Theorem $\mathrm{G}$ and proved that the following is true.

Theorem M. Let $f \in L_{1}(\mathbb{I})$ and

$$
\sup _{A} \frac{1}{\left|m_{A}\right|} \sum_{k=1}^{\left|m_{A}\right|} \varepsilon_{k}\left(m_{A}\right) l_{m_{A}(k-1)}<\infty .
$$

Then $t_{m_{A}}(x, f) \rightarrow f(x)$ as $A \rightarrow \infty$ for a. e. $x \in \mathbb{I}$.

In this paper we are going to replace condition (4.2) with more weaker condition

$$
\sup _{A} \frac{1}{\left|m_{A}\right|} \sum_{k=1}^{\left|m_{A}\right|}\left|\varepsilon_{k}\left(m_{A}\right)-\varepsilon_{k+1}\left(m_{A}\right)\right| l_{m_{A}(k-1)}<\infty .
$$

It is easy to see that condition (4.2) imply condition (4.3), on other hand, for the sequence $\left\{2^{A}-1: A \in \mathbb{N}\right\}$ condition (4.2) does not holds and condition (4.3) holds. So, we prove that the following is valid.

Theorem 2. Let $f \in L_{1}(\mathbb{I})$ and condition (4.3) is holds. Then $t_{m_{A}}(x, f) \rightarrow f(x)$ as $A \rightarrow \infty$ for a. e. $x \in \mathbb{I}$.

Proof. From (3.4) we have

$$
f *\left(l_{m_{A}} F_{m_{A}}\right)(x)=f * H_{m_{A}}^{(1)}(x)+f * H_{m_{A}}^{(2)}(x)+f * H_{m_{A}}^{(3)}(x) .
$$

It is easy to see that

$$
\left\|\sup _{A}\left|f * H_{m_{A}}^{(3)}\right|\right\|_{1} \lesssim\|f\|_{1} .
$$

From (3.5) we can write

$$
f * H_{m_{A}}^{(2)}(x)=f * H_{m_{A}}^{(21)}(x)+f * H_{m_{A}}^{(22)}(x)+f * H_{m_{A}}^{(22)}(x) .
$$

Using (1.1) we have

$$
\left|f * H_{m_{A}}^{(21)}(x)\right| \leq \sum_{j=1}^{\left|m_{A}\right|-1}\left(|f| * D_{2^{j}}(x)\right) \leq\left|m_{A}\right| E^{*}(x, f) .
$$


Hence

$$
\sup _{A} \frac{\left|f * H_{m_{A}}^{(21)}(x)\right|}{\left|m_{A}\right|} \leq E^{*}(x, f) .
$$

We can write

$$
\begin{aligned}
\left|f * H_{m_{A}}^{(22)}(x)\right| \leq & \sum_{j=1}^{\left|m_{A}\right|-1} \varepsilon_{j}\left(m_{A}\right)\left(l_{m_{A}(j)}-l_{m_{A}(j-1)}\right)\left(|f| * D_{2^{j}}(x)\right) \\
& \lesssim\left|m_{A}\right| E^{*}(x, f), \\
& \sup _{A} \frac{\left|f * H_{m_{A}}^{(22)}(x)\right|}{\left|m_{A}\right|} \lesssim E^{*}(x, f) .
\end{aligned}
$$

It is proved in [7]

$$
\sup _{\lambda} \lambda\left|\left\{\sup _{A} \frac{\left|f * H_{m_{A}}^{(23)}\right|}{\left|m_{A}\right|}>\lambda\right\}\right| \lesssim\|f\|_{1} .
$$

Since $\sup \lambda\left|\left\{E^{*}(f)>\lambda\right\}\right| \lesssim\|f\|_{1}$ from (4.7)- (4.9) we get

$$
\sup _{\lambda} \lambda\left|\left\{\sup _{A} \frac{\left|f * H_{m_{A}}^{(2)}\right|}{\left|m_{A}\right|}>\lambda\right\}\right| \lesssim\|f\|_{1} .
$$

Now, we estimate $\left|f * H_{m_{A}}^{(1)}(x)\right|$. We have

$$
\begin{aligned}
\left|f * H_{m_{A}}^{(1)}(x)\right| \lesssim & \sum_{j=1}^{\left|m_{A}\right|-1}\left(\varepsilon_{j}\left(m_{A}\right) l_{m_{A}(j-1)}-\varepsilon_{j+1}\left(m_{A}\right) l_{m_{A}(j)}\right)\left(|f| * D_{2^{j}}(x)\right) \\
& +l_{\left(\left|m_{A}\right|-1\right)}\left(|f| * D_{2}\left|m_{A}\right|+1\right. \\
\lesssim & E^{*}(x, f)\left(l_{\left(\left|m_{A}\right|-1\right)}+\sum_{j=1}^{\left|m_{A}\right|-1}\left(\varepsilon_{j}\left(m_{A}\right) l_{m_{A}(j-1)}-\varepsilon_{j+1}\left(m_{A}\right) l_{m_{A}(j)}\right)\right) \\
\lesssim & E^{*}(x, f)\left(l_{\left(\left|m_{A}\right|-1\right)}+\sum_{j=1}^{\left|m_{A}\right|-1}\left|\varepsilon_{j}\left(m_{A}\right)-\varepsilon_{j+1}\left(m_{A}\right)\right| l_{m_{A}(j-1)}\right. \\
& \left.+\sum_{j=1}^{\left|m_{A}\right|-1} \varepsilon_{j+1}\left(m_{A}\right)\left(l_{m_{A}(j)}-l_{m_{A}(j-1)}\right)\right)
\end{aligned}
$$




$$
\lesssim E^{*}(x, f)\left(l_{\left(\left|m_{A}\right|-1\right)}+\sum_{j=1}^{\left|m_{A}\right|-1}\left|\varepsilon_{j}\left(m_{A}\right)-\varepsilon_{j+1}\left(m_{A}\right)\right| l_{m_{A}(j-1)}\right) .
$$

From the condition of the Theorem we can write

$$
\sup _{A} \frac{\left|f * H_{m_{A}}^{(1)}(x)\right|}{l_{m_{A}}} \lesssim E^{*}(x, f) V_{L}\left(m_{A}\right)
$$

and

$$
\sup _{\lambda} \lambda\left|\left\{\sup _{A} \frac{\left|f * H_{m_{A}}^{(1)}\right|}{\left|m_{A}\right|}>\lambda\right\}\right| \lesssim\|f\|_{1} .
$$

Combining (3.5), (4.5), (4.10) and (4.11) we conclude that

$$
\sup _{\lambda} \lambda\left|\left\{\sup _{A} \frac{\left|f * F_{m_{A}}\right|}{\left|m_{A}\right|}>\lambda\right\}\right| \lesssim\|f\|_{1} .
$$

By the well-known density argument we complete the proof of Theorem 2.

\section{UNIFORM AND $L$-CONVERGENCE OF LOGARITHMIC MEANS}

Denote by $C_{w}(\mathbb{I})$ the space of uniformly continuous functions on $\mathbb{I}$, with the supremum norm

$$
\|f\|_{C_{w}}:=\sup _{x \in \mathbb{I}}|f(x)| \quad\left(f \in C_{w}(\mathbb{I})\right) .
$$

Let $X=X(\mathbb{I})$ be either the space $L_{1}(\mathbb{I})$, or the space of uniformly continuous functions, that is, $C_{w}(\mathbb{I})$. The corresponding norm is denoted by $\|\cdot\|_{X}$.

For Walsh-Fourier series Fine [2] has obtained a sufficient condition for the uniform convergence which is in a complete analogy with the Dini-Lipshitz condition (see also [20]). Similar results are true for the space of integrable functions $L_{1}$ (II) [19]. Gulicev [13] has estimated the rate of uniform convergence of a Walsh-Fourier series using Lebesgue constant and modulus of continuity. Uniform convergence of Walsh-Fourier series of the functions of classes of generalized bounded variation was investigated by author [10]. This problem has been considered for Vilenkin group by Fridli [3] and Gát [5]. Lukomskii [15] considered uniform and $L_{1}$-convergence of subsequence of partial sums of Walsh-Fourier series. In particular, he proved that the condition $\sup V_{S}\left(m_{A}\right)<\infty$ is necessary and sufficient condition for uniform and

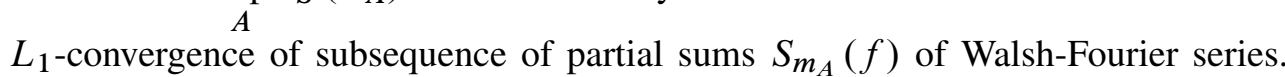
In Móricz and Siddiqi [17] investigated approximation properties of Nörlund means $\frac{1}{Q_{n}} \sum_{k=0}^{n-1} q_{n-k} S_{k} f$. The case when we have $q_{k}:=1 / k$ differs from the types discussed by Móricz and Siddiqi in [17]. His method is not applicable for logarithmic 
means. In [6] it is proved that Theorem of Móricz does not hold for $L_{1}, C_{w}$ and $q_{k}:=1 / k$.

In [12] it is investigated $X$-norm convergence of subsequence of logarithmic means of Walsh-Fourier series. In particular, the following are proved.

Theorem GT. a) Let $f \in X$ (I) and

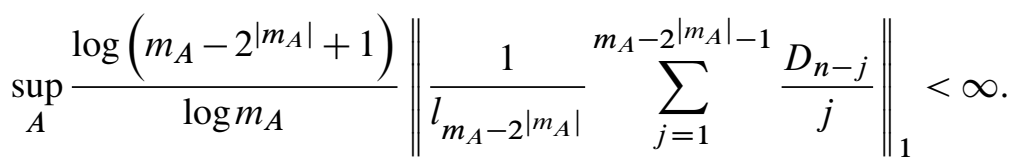

Then subsequence of Nörlund logarithmic means $t_{m_{A}}(f)$ converges in the norm of space $X(\mathbb{I})$.

b) If the condition (5.1) does not holds then we can find a function from the space $X$ (II) for which the convergence of logarithmic means $L_{m_{A}}(f)$ in the norm of space $X$ (II) does not holds.

Since

$$
\begin{aligned}
& \sup _{A} \frac{\log \left(m_{A}-2^{\left|m_{A}\right|}+1\right)}{\log m_{A}}\left\|\frac{1}{l_{m_{A}-2}\left|m_{A}\right|} \sum_{j=1}^{m_{A}-2^{\left|m_{A}\right|}-1} \frac{D_{n-j}}{j}\right\|_{1} \\
& \sim \sup _{A} \frac{\log \left(m_{A}-2^{\left|m_{A}\right|}+1\right)}{\log m_{A}} \frac{1}{\left|m_{A}-2^{\left|m_{A}\right|}\right|} \sum_{k=1}^{\left|m_{A}-2^{\left|m_{A}\right|}\right|}\left|\varepsilon_{k}(n)-\varepsilon_{k+1}(n)\right| l_{n(k-1)} \\
& \sim \sup _{A} \frac{1}{\left|m_{A}\right|} \sum_{k=1}^{\left|m_{A}\right|}\left|\varepsilon_{k}(n)-\varepsilon_{k+1}(n)\right| l_{n(k-1)},
\end{aligned}
$$

from Theorem GT and Theorem 1 we can prove necessary and sufficint condition for norm convergence of subsequence of Nörlund logarithmic means

Theorem 3. Let $f \in X(\mathbb{I})$. Then the condition $\sup V_{L}\left(m_{A}\right)<\infty$ is neccessary and sufficient for convergence subsequence of Nörlund logarithmic means of WalshFourier series in norm of space $X(\mathbb{I})$.

\section{REFERENCES}

[1] I. Blahota and G. Gát, "Norm summability of Nörlund logarithmic means on unbounded Vilenkin groups," Anal. Theory Appl., vol. 24, no. 1, pp. 1-17, 2008, doi: 10.1007/s10496-008-0001-z. [Online]. Available: https://doi.org/10.1007/s10496-008-0001-z

[2] N. J. Fine, "On the Walsh functions," Trans. Amer. Math. Soc., vol. 65, pp. 372-414, 1949, doi: 10.2307/1990619. [Online]. Available: https://doi.org/10.2307/1990619 
[3] S. Fridli, "Approximation by Vilenkin-Fourier sums," Acta Math. Hungar, vol. 47, no. 1-2, pp. 33-44, 1986, doi: 10.1007/BF01949122. [Online]. Available: https: //doi.org/10.1007/BF01949122

[4] G. Gát, "Investigations of certain operators with respect to the Vilenkin system," Acta Math. Hungar., vol. 61, no. 1-2, pp. 131-149, 1993, doi: 10.1007/BF01872107. [Online]. Available: https://doi.org/10.1007/BF01872107

[5] G. Gát, "Best approximation by Vilenkin-like systems," Acta Math. Acad. Paedagog. Nyházi. (N.S.), vol. 17, no. 3, pp. 161-169, 2001.

[6] G. Gát and U. Goginava, "Uniform and $L$-convergence of logarithmic means of WalshFourier series," Acta Math. Sin. (Engl. Ser.), vol. 22, no. 2, pp. 497-506, 2006, doi: 10.1007/s10114-005-0648-8. [Online]. Available: https://doi.org/10.1007/s10114-005-0648-8

[7] G. Gát and U. Goginava, "On the divergence of Nörlund logarithmic means of WalshFourier series," Acta Math. Sin. (Engl. Ser.), vol. 25, no. 6, pp. 903-916, 2009, doi: 10.1007/s10114-009-7013-2. [Online]. Available: https://doi.org/10.1007/s10114-009-7013-2

[8] G. Gát and U. Goginava, "Norm convergence of logarithmic means on unbounded Vilenkin groups," Banach J. Math. Anal., vol. 12, no. 2, pp. 422-438, 2018, doi: 10.1215/17358787-20170031. [Online]. Available: https://doi.org/10.1215/17358787-2017-0031

[9] G. Gát, U. Goginava, and G. Tkebuchava, "Convergence of logarithmic means of multiple WalshFourier series," Anal. Theory Appl., vol. 21, no. 4, pp. 326-338, 2005, doi: 10.1007/BF02836855. [Online]. Available: https://doi.org/10.1007/BF02836855

[10] U. Goginava, "On the uniform convergence of Walsh-Fourier series," Acta Math. Hungar. vol. 93, no. 1-2, pp. 59-70, 2001, doi: 10.1023/A:1013865315680. [Online]. Available: https://doi.org/10.1023/A:1013865315680

[11] U. Goginava, "Almost everywhere convergence of subsequence of logarithmic means of WalshFourier series," Acta Math. Acad. Paedagog. Nyházi. (N.S.), vol. 21, no. 2, pp. 169-175, 2005.

[12] U. Goginava and G. Tkebuchava, "Convergence of subsequences of partial sums and logarithmic means of Walsh-Fourier series," Acta Sci. Math. (Szeged), vol. 72, no. 1-2, pp. 159-177, 2006.

[13] N. V. Guličev, "Approximation to continuous functions by Walsh-Fourier sums," Anal. Math., vol. 6, no. 4, pp. 269-280, 1980, doi: 10.1007/BF02053633. [Online]. Available: https://doi.org/10.1007/BF02053633

[14] S. V. Konyagin, "On a subsequence of Fourier-Walsh partial sums," Mat. Zametki, vol. 54, no. 4, pp. 69-75, 158, 1993, doi: 10.1007/BF01210421. [Online]. Available: https://doi.org/10.1007/BF01210421

[15] S. F. Lukomskiü, "Multiple Walsh series. Convergence in measure and almost everywhere," Dokl. Akad. Nauk, vol. 358, no. 4, pp. 459-460, 1998.

[16] N. Memić, "Almost everywhere convergence of some subsequences of the Nörlund logarithmic means of Walsh-Fourier series," Anal. Math., vol. 41, no. 1-2, pp. 45-54, 2015, doi: 10.1007/s10476-015-0104-7. [Online]. Available: https://doi.org/10.1007/s10476-015-0104-7

[17] F. Móricz and A. H. Siddiqi, "Approximation by Nörlund means of Walsh-Fourier series," J. Approx. Theory, vol. 70, no. 3, pp. 375-389, 1992, doi: 10.1016/0021-9045(92)90067-X. [Online]. Available: https://doi.org/10.1016/0021-9045(92)90067-X

[18] K. Nagy, "Almost everywhere convergence of a subsequence of the Nörlund logarithmic means of Walsh-Kaczmarz-Fourier series," J. Math. Inequal., vol. 3, no. 4, pp. 499-510, 2009, doi: 10.7153/jmi-03-49. [Online]. Available: https://doi.org/10.7153/jmi-03-49

[19] C. W. Onneweer, "On $L_{1}$-convergence of Walsh-Fourier series," Internat. J. Math. Math. Sci., vol. 1, no. 1, pp. 47-56, 1978, doi: 10.1155/S016117127800006X. [Online]. Available: https://doi.org/10.1155/S016117127800006X

[20] F. Schipp, W. R. Wade, and P. Simon, Walsh series. Adam Hilger, Ltd., Bristol, 1990, an introduction to dyadic harmonic analysis, With the collaboration of J. Pál. 
[21] P. Simon, "Strong convergence of certain means with respect to the Walsh-Fourier series," Acta Math. Hungar., vol. 49, no. 3-4, pp. 425-431, 1987, doi: 10.1007/BF01951006. [Online]. Available: https://doi.org/10.1007/BF01951006

\section{Author's address}

\section{Ushangi Goginava}

U. Goginava, I. Vekua Institute of Applied Mathematics and Faculty of Exact and Natural Sciences of I. Javakhishvili Tbilisi State University, Tbilisi 0186, 2 University str., Georgia

E-mail address: zazagoginavaegmail.com 